\title{
A novel strategy to generate virus vaccines with expanded genetic codes
}

\author{
Deyin Guo ${ }^{1,2 *}$ \\ ${ }^{1}$ School of Basic Medicine (Shenzhen), Sun Yat-sen University, Guangdong 510080, China; \\ ${ }^{2}$ School of Basic Medical Sciences, Wuhan University, Wuhan 430072, China
}

Received December 28,2016; accepted January 17, 2017; published online March 13, 2017

Citation: Guo, D. (2017). A novel strategy to generate virus vaccines with expanded genetic codes. Sci China Life Sci 60 , 555-557. doi: 10.1007/s11427-0169010-y

The invention and application of vaccines for combating infectious diseases represent one of the greatest human accomplishments within the medical and health sectors in the history of mankind. The smallpox virus, once devastating and widespread across the continents, is the first and sole human pathogen that has been eradicated globally, and this great success is attributed to the use of vaccinia vaccines. Efficacious vaccines have been developed and applied successfully for the prevention of a number of infectious viral diseases, such as smallpox, poliomyelitis, measles, mumps and rubella (Hajj Hussein et al., 2015). However, substantial challenges remain in obtaining safe and efficacious vaccines for a variety of newly emerging and re-emerging viruses, such as the severe acute respiratory syndrome coronavirus (SARS-CoV), Middle East respiratory syndrome (MERS)-CoV, Ebola virus, rotavirus, H5N1 and H7N9 influenza, and Dengue and Zika viruses, as well as chronic infections, such as human immunodeficiency virus (HIV) and hepatitis $\mathrm{C}$ virus (HCV). In the field of vaccine development, there is also a concern that live attenuated vaccines could revert to their pathogenic forms. Therefore, great efforts have been made in recent decades to develop new strategies for vaccine generation and to improve vaccine efficacy and safety. In a very recent work by Dr. Demin

*Corresponding author (email: dguo@whu.edu.cn)
Zhou and his research team at Peking University, a novel strategy using expanded genetic codes was developed to directly convert fully infectious influenza virus into avirulent, replication-deficient vaccines by inserting premature termination codons (PTCs) into the viral genome (Si et al., 2016). Similar strategies were previously adopted for generating live-attenuated HIV-1 vaccine (Wang et al., 2014) and modifying hepatitis D virus (Lin et al., 2013).

Protein translation machinery normally reads the 61 genetic triplet codons of mRNAs as corresponding to 20 standard amino acids; three codons (UAG, UAA and UGA) are stop signals. However, the genetic code is not $100 \%$ universal in organisms of distinct lineages. For example, in some prokaryotic cells, the UAG nonsense codon (nicknamed the amber codon) can be recognized as a sense code for pyrrolysine, one of the noncanonical amino acids outside of the standard 20-member amino acid alphabet (Chin et al., 2003). Si and colleagues cleverly utilized the diversity of codon assignments to modify the viral genomes and engineer mammalian cells for virus packaging and propagation (Si et al., 2016). They replaced 1-8 sense codons with UAG codons in the eight RNA genomic segments of the influenza A virus (IAV) and inserted genes coding for the orthogonal translation components of Methanosarcina barkeri, namely, pyrrolysyl tRNA synthetase (MbpylRS) and tRNA ${ }_{\mathrm{CUA}}$, into the chromosomes of human embryonic kidney (HEK) 293T cells. As expected, the recombinant PTC-har- 
bouring IAV can efficiently replicate only in the genetically modified packaging cells bearing the Mbpyl/tRNA ${ }_{\text {CUA }}$ genes in the presence of unnatural pyrrolysine (UPL) and not in conventional human cells.

The efficacy of the PTC viruses as live vaccines was tested in mice, guinea pigs and ferrets. One recombinant influenza virus harbouring 4 PTCs (PTC-4A virus) elicited robust mucosal, humoral and cell-mediated immunity and protected the animals from significant loss of body weight and death to a similar extent as the commercially available attenuated influenza vaccine and was apparently superior to the inactivated vaccine. More excitingly, the PTC-4A virus could induce cross-protection against antigenically distant influenza strains and exert inhibitory effects on co-infecting wild-type viruses in conventional cells. Genetic reassortment was observed between the PTC virus and other co-existing viruses, resulting in PTC-harbouring replication-incompetent reassortants, which may be the cause of the neutralization of virulent wild-type viruses. This observation indicates that the PTC virus vaccine may have potential therapeutic effects in addition to its prophylactic potency.

While this was a proof-of-principle study, this landmark work provided a smart, innovative approach to generating effective live vaccines that are replication-competent in engineered cells but replication-deficient and infection-capable in conventional cells. This novel type of PTC live vaccines may have several advantages over the classical attenuated live vaccines (CALV). First, the generation of PTC vaccines is fast and convenient if the genome of a virus is known and cloned. In contrast, the creation of CALV, which is normally prepared through a series of passages and virulence tests in cell cultures and animal hosts, is time-consuming. Second, the PTC vaccines may be more immunogenic and more efficacious than CALV because the former can maintain full antigenicity and infectivity, while CALV could be compromised after the many passages to achieve virulence attenuation. Third, the PTC vaccines might be biologically safer than CALV. The PTCs are nonsense stop codons and thus are non-translatable in conventional cells and humans; therefore, the PTC-bearing viruses may have a lower chance of reverting to replication-competent pathogenic forms, suggesting that the PTC viruses may have a higher level of safety than conventional attenuated live vaccines. Fourth, the approach for PTC vaccines is universal and may be applied to other viruses, including the ones that urgently need effective vaccines, such as Ebola, MERS, Zika, Marburg and Chikungunya. Indeed, the PTC virus has been generated for HIV-1 (Wang et al., 2014).

The major concerns about live vaccines are the possible reversion to virulent forms through mutation and selection pressure during replication or the generation of more virulent strains through recombination and reassortment with naturally circulating viruses during infection; the PTC virus vaccines are not exempt from these concerns. As shown by $\mathrm{Si}$ et al., the PTC influenza viruses could revert their UAG codons to sense codons and thus escape the PTC constraints during replication and propagation at varying frequencies depending on the location and number of the UAG codons (Si et al., 2016). Therefore, to make safe PTC vaccines, careful selection and multiple insertions of UAG codons are essential. Luckily, revertant influenza viruses were not detected in animal models, and this may be ascribed to the PTC vaccine-induced potent immune response and the fast clearance of rare revertant viruses by the immune system. The PTC influenza viruses could also reassort with co-infecting wild-type viruses. Surprisingly, such reassortment decreased the fitness and propagation of the co-existing viruses. However, the effect of the reassortment on virus fitness and pathogenicity should be further tested in animal models. Although the possibility of reversion to virulence cannot be excluded for PTC vaccines, they may still have a higher level of biological safety than conventional attenuated live vaccines.

The first use of vaccination, i.e., variolation to prevent smallpox, dates back to 1,000 A.D. in China. Since the first laboratory vaccine, the live attenuated rabies vaccine, was made by Louis Pasteur in the late nineteenth century, the techniques for preparing efficacious and safe vaccines have been improving constantly. The two major types of vaccines used in the current immunization scheme are inactivated whole virus vaccines and live attenuated vaccines. Since the advent of recombinant DNA technology, new vaccine strategies have been explored as alternatives to classical vaccines, and they include recombinant viral protein and synthetic peptides, virus-like particles and nanoparticles, avirulent virus-vectored vaccines, and DNA and RNA vaccines (García-Sastre and Mena, 2013). Although these types of vaccines may appear to be safer, they are also diminished in immunogenicity and efficacy. The strategy reported by the Chinese group led by Dr. Zhou provides a completely new tool for the preparation of immunologically effective influenza vaccines (Si et al., 2016). This represents a breakthrough in the vaccine field and may revolutionize vaccine development, especially against emerging and re-emerging RNA viruses. The amber codons are used as PTCs in the new type of influenza vaccines. Coincidentally, the amber gemstone is usually regarded as a lucky stone and a good omen among many cultures in the world. It may be hoped that the amber-bearing PTC vaccines can be further developed in clinical trials and that their successful application may greatly benefit global public health by preventing the infection of life-threatening viruses.

Compliance and ethics The author(s) declare that they have no conflict of interest.

Chin, J.W., Ashton Cropp, T., Anderson, J.C., Mukherji, M., Zhang, Z., and 
Schultz, P.G. (2003). An expanded eukaryotic genetic code. Science 301, 964-967.

García-Sastre, A., and Mena, I. (2013). Novel vaccine strategies against emerging viruses. Curr Opin Virol 3, 210-216.

Hajj Hussein, I., Chams, N., Chams, S., El Sayegh, S., Badran, R., Raad, M., Gerges-Geagea, A., Leone, A., and Jurjus, A. (2015). Vaccines through centuries: major cornerstones of global health. Front Public Health 3, 269.

Lin, S., Yan, H., Li, L., Yang, M., Peng, B., Chen, S., Li, W., and Chen,
P.R. (2013). Site-specific engineering of chemical functionalities on the surface of live hepatitis D virus. Angew Chem Int Ed 52, 13970-13974. Si, L., Xu, H., Zhou, X., Zhang, Z., Tian, Z., Wang, Y., Wu, Y., Zhang, B., Niu, Z., Zhang, C., Fu, G., Xiao, S., Xia, Q., Zhang, L., and Zhou, D. (2016). Generation of influenza A viruses as live but replication-incompetent virus vaccines. Science 354, 1170-1173.

Wang, N., Li, Y., Niu, W., Sun, M., Cerny, R., Li, Q., and Guo, J. (2014). Construction of a live-attenuated HIV-1 vaccine through genetic code expansion. Angew Chem Int Ed 53, 4867-4871. 